\title{
Making waves in physics
}

\section{Three wave singularities from the miraculous 1830 s.}

\section{Michael Berry}

$\mathrm{S}$ ingularities are places where mathematical quantities become infinite, or change abruptly. In waves - of light, for example - there can be singularities in the intensity, in the phase, or in the polarization. This is a modern view, sharply different from the traditional approach where waves were simply the solutions of wave equations, and singularities - if considered at all were regarded as awkward places where the usual treatments fail. And yet the foundations of wave singularities were laid in three astonishing papers published as early as the 1830 s, a decade whose intellectual significance we are only now beginning to appreciate.

Reversing historical order, we start in 1838 , with a paper by George Biddell Airy. The immediate stimulus was the theory of the rainbow. Two centuries earlier, Descartes had understood the bright bow as Sun rays directionally focused by raindrops. This geometrical theory gives a good first approximation but fails to account for the delicate supernumerary bows sometimes seen just inside the main arc. In 1801 Thomas Young realized that by regarding light as waves it is possible to understand supernumaries as interference fringes, but could not give a precise mathematical description. Airy's contributions were: to appreciate that the rainbow is a particular example of a caustic, that is, a line where light rays are focused (the bright lines on the bottom of swimming pools are also caustics); to see that caustics are singularities, where ray optics predicts infinite brightness; to realize that wave physics will soften the singularities; and to discover the precise mathematical description of this softening, in the form of his rainbow integral (pictured above).

Airy's paper was doubly influential. First, because refined techniques soon devised by George Gabriel Stokes to study the rainbow integral established divergent infinite series as an important tool in bridging gaps between physical theories (in this case ray and wave optics) and uncovered a mathematical phenomenon whose ramifications are still being explored. Second, because the rainbow integral, describing wave interference decorating the simplest kind of caustic, was found to be the first in a hierarchy of 'diffraction catastrophes'. These more elaborate wave singularities are now classified using catastrophe theory - mathematics whose application greatly advances the physics of caustics.

Next are two papers from 1833 and 1836 by the polymath William Whewell (to whom

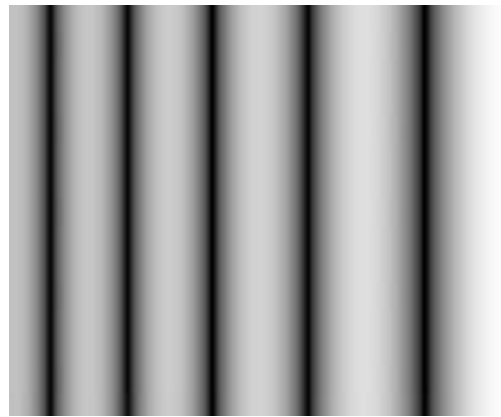

Airy's rainbow integral.

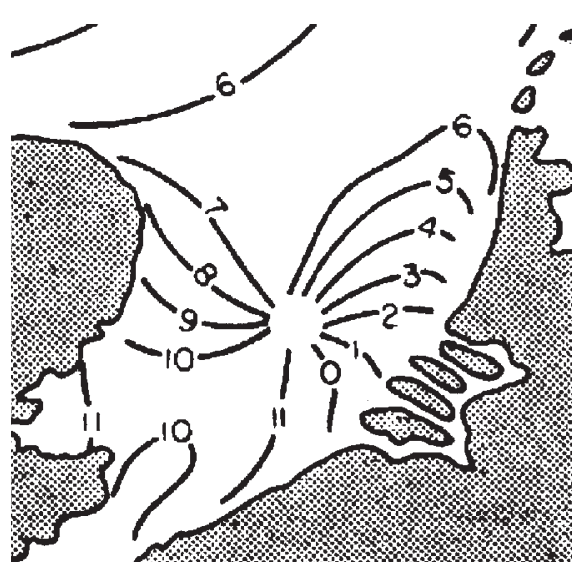

Whewell's amphidromy between England and Holland.

we owe the word 'physicist', with its "four sibilant consonants that fizz like a squib"). He was studying the tides in the oceans, and seeking to "connect the actual tides of all the different parts of the world — and to account for their varieties and seeming anomalies". From Young he learned to concentrate on the cotidal lines connecting places where the tide is high at a given time. Cotidal lines are wavefronts of the tide, regarded as a wave of twelve-hour period; they are contours of the phase of this wave. Whewell appreciated that a map of cotidal lines would render intelligible the pattern of tides around the coasts, and coordinated hundreds of new observations, in an early international scientific collaboration.

Extrapolating away from the coasts into the 'German Ocean' he reached the extraordinary conclusion that there must be "rotatory systems of tide-waves [where] the cotidal lines ... revolve around [a point] where there is no tide, for it is clear that at a point where all the cotidal lines meet, it is high-water equally at all times". One of Whewell's 'amphidromies' can be seen above. What Whewell discovered were the phase singularities of the tide waves.

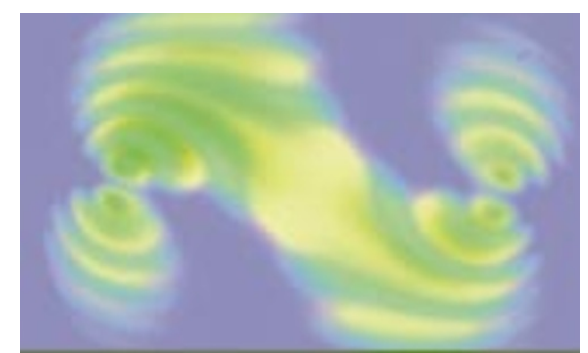

Hamilton's diabolical points (bullseyes) in several square centimetres of overhead-projector transparency foil viewed obliquely through crossed polarizers; in each bullseye, the interference rings are contours of difference of wave speeds, centred on an optic axis, and the black stripes reflect geometric phases.

Phase singularities are now recognized as important features of all waves; in three dimensions they are lines rather than points. At a phase singularity, the wave intensity is zero - in contrast to caustics where the intensity is (geometrically) infinite. In acoustics, the singularities are threads of silence; in light, they are optical vortices; in superfluids, quantized vortices; and in superconductors, quantized lines of magnetic flux.

In addition to intensity and phase, waves of light exhibit polarization: they are vector waves. Polarization has its singularities too, discovered by our third author, William Rowan Hamilton, in 1832, as an unexpected consequence of Augustin Fresnel's theory of the optics of crystals. In a general anisotropic material, two waves propagate in each direction, with different speeds and polarizations. There are, however, two singular directions, optic axes, where the speeds are the same. As functions of direction, the two speeds can be represented by surfaces forming a diabolo (double cone) at each optic axis. Hamilton deduced that at such 'diabolical points' the wave direction corresponds to a cone of rays. This unprecedented 'conical refraction' was soon observed, confirming that light is a transverse wave. Diabolical points can easily be seen directly (pictured above).

Hamilton's diabolical point was the first physical example of degeneracy between eigenvalues of a real symmetric matrix. Its descendants thrive in many areas of science today, for example, as conical intersections between energy levels in quantum chemistry, as Bloch wave degeneracies in the quantum Hall effect, and as the simplest geometric phases.

Michael Berry is in the Department of

Physics, University of Bristol, Bristol

BS8 1TL, UK. 